\title{
Biharmonic and Quasi-Biharmonic Slant Surfaces in Lorentzian Complex Space Forms
}

\begin{abstract}
Yu Fu
School of Mathematics and Quantitative Economics, Dongbei University of Finance and Economics, Dalian 116025, China

Correspondence should be addressed to Yu Fu; yu_fu@yahoo.cn

Received 6 December 2012; Accepted 12 March 2013

Academic Editor: Jaeyoung Chung

Copyright (C) $2013 \mathrm{Yu} \mathrm{Fu}$. This is an open access article distributed under the Creative Commons Attribution License, which permits unrestricted use, distribution, and reproduction in any medium, provided the original work is properly cited.

In 1991, Chen and Ishikawa initially studied biharmonic marginally trapped surfaces in neutral pseudo-Euclidean 4-space. Recently, biharmonic and quasi-biharmonic marginally trapped Lagrangian surfaces in Lorentzian complex space forms were studied by Sasahara in 2007 and 2011, respectively. In this paper we extend Sasahara's results to the case of slant surfaces in Lorentzian complex space forms. By results, we completely classify biharmonic marginally trapped slant surfaces and quasi-biharmonic marginally trapped slant surfaces in Lorentzian complex space forms.
\end{abstract}

\section{Introduction}

Let $\widetilde{M}_{i}^{n}(4 c)$ be a simply connected Lorentzian complex space form of complex dimension $n$ and complex index $i(i \geq 0)$, where the complex index is defined as the complex dimension of the largest complex negative definite subspace of the tangent space. In particular, if $i=1$, we say that $\widetilde{M}_{1}^{n}(4 c)$ is Lorentzian. The curvature tensor $\widetilde{R}$ of $\widetilde{M}_{i}^{n}(4 c)$ is given by

$$
\begin{array}{r}
\widetilde{R}(X, Y) Z=c\{\langle Y, Z\rangle X-\langle X, Z\rangle Y+\langle J Y, Z\rangle J X \\
-\langle J X, Z\rangle J Y+2\langle X, J Y\rangle J Z\} .
\end{array}
$$

Let $\mathbb{C}^{n}$ denote the complex number $n$-space with complex coordinates $z_{1}, \ldots, z_{n}$. The $\mathbb{C}^{n}$ endowed with $g_{s, n}$, that is, the real part of the Hermitian form

$$
b_{s, n}(z, \omega)=-\sum_{k=1}^{s} \bar{z}_{k} \omega_{k}+\sum_{j=s+1}^{n} \bar{z}_{j} \omega_{j}, \quad z, \omega \in \mathbb{C}^{n},
$$

defines a flat indefinite complex space form with complex index $s$. Denote the pair $\left(\mathbb{C}^{n}, g_{s, n}\right)$ by $\mathbb{C}_{s}^{n}$ briefly, which is the flat Lorentzian complex $n$-space. In particular, $\mathbb{C}_{1}^{2}$ is the flat Lorentzian complex plane.

Let us consider the differentiable manifold:

$$
S_{2}^{2 n+1}(c)=\left\{z \in \mathbb{C}_{1}^{n+1} ; b_{1, n+1}(z, z)=c^{-1}>0\right\},
$$

which is an indefinite real space form of constant sectional curvature $c$. The Hopf fibration

$$
\pi: S_{2}^{2 n+1}(c) \longrightarrow C P_{1}^{n}(4 c): z \longmapsto z \cdot \mathbb{C}^{*}
$$

is a submersion and there exists a unique pseudo-Riemannian matrix of complex index one on $C P_{1}^{n}(4 c)$ such that $\pi$ is a Riemannian submersion.

The pseudo-Riemannian manifold $C P_{1}^{n}(4 c)$ is a Lorentzian complex space form of positive holomorphic sectional curvature $4 c$.

Analogously, if $c<0$, consider

$$
H_{2}^{2 n+1}(c)=\left\{z \in \mathbb{C}_{2}^{n+1} ; b_{1, n+1}(z, z)=c^{-1}<0\right\},
$$

which is an indefinite real space form of constant sectional curvature $c$. The Hopf fibration

$$
\pi: H_{2}^{2 n+1}(c) \longrightarrow \mathrm{CH}_{1}^{n}(4 c): z \longmapsto z \cdot \mathbb{C}^{*}
$$

is a submersion and there exists a unique pseudo-Riemannian matrix of complex index one on $\mathrm{CH}_{1}^{n}(4 c)$ such that $\pi$ is a Riemannian submersion.

The pseudo-Riemannian manifold $\mathrm{CH}_{1}^{n}(4 c)$ is a Lorentzian complex space form of negative holomorphic sectional curvature $4 c$.

It is well known that a complete simply connected complex space form $\widetilde{M}_{i}^{n}(4 c)$ is holomorphic isometric to $\mathbb{C}_{1}^{n}$, 
$C P_{1}^{n}(4 c)$, or $C H_{1}^{n}(4 c)$, according to $c=0, c>0$, or $c<0$, respectively.

A real surface in a Kähler surface with almost complex structure $J$ is called slant if its Wirtinger angle is constant (see [1-3]). From $J$-action point of views, slant surfaces are the simplest and the most natural surfaces of a Lorentzian Kähler surface $(\widetilde{M}, \widetilde{g}, J)$. It should be pointed out that slant surfaces arise naturally and play important roles in the studies of surfaces of Kähler surfaces in the complex space forms; see [4].

In last years, the geometry of Lorentzian surfaces in Lorentzian complex space forms has been studied by a series of papers given by Chen and other geometers, for instance, $[1,3,5-13]$. Lorentzian geometry is a vivid field of mathematical research that represents the mathematical foundation of the general theory of relativity-which is probably one of the most successful and beautiful theories of physics. For Lorentzian surfaces immersed in Lorentzian complex space forms, Chen [7] proved that Ricci equation is a consequence of Gauss and Codazzi equations, which indicates that Lorentzian surfaces in Lorentzian complex space forms have many interesting properties.

During the last decade, the theory of biharmonic submanifolds has advanced greatly. By definition, a submanifold is called biharmonic if the bitension field of the isometric immersion defining the submanifold vanishes identically. There are a lot classification results and nonexistence results, (see, e.g., $[4,14,15])$. Recently, Sasahara introduces the notion of quasi-biharmonic submanifold in [16], which is defined with the property that the bitension field of the isometric immersion defining the submanifold is lightlike at each point. It is shown in [16] that the class of quasi-biharmonic submanifolds is quite different from the class of biharmonic submanifolds.

A surface of a pseudo-Riemannian manifold is called marginally trapped (or quasiminimal) if its mean curvature vector field is lightlike. In the theory of cosmic black holes, a marginally trapped surface in a space-time plays an extremely important role. From the viewpoint of differential geometry, some classification results on marginally trapped surfaces have been obtained by some geometers (see $[1,3,9-12]$ ). In particular, Chen and Dillen [9] gave a complete classification of marginally trapped Lagrangian surfaces in Lorentzian complex space forms.

In this paper, we investigate the bitension field of marginally trapped slant surfaces in Lorentzian complex space forms. In particular, we completely classify biharmonic marginally trapped slant surfaces and quasi-biharmonic marginally trapped slant surfaces in Lorentzian complex space forms, respectively (see Theorems 12 and 13). Our classification results extend Sasahara's results from Lagrangian case to the slant case in Lorentzian complex space forms.

\section{Preliminaries}

2.1. Basic Notation, Formulas, and Definitions. Let $M$ be a Lorentzian surface of a Lorentzian Kähler surface $\widetilde{M}_{1}^{2}$ equipped with an almost structure $J$ and metric $\tilde{g}$. Let $\langle$, denote the inner product associated with $\widetilde{g}$.

We denote the Levi-Civita connections of $M$ and $\widetilde{M}_{1}^{2}$ by $\nabla$ and $\widetilde{\nabla}$, respectively. Gauss formula and Weingarten formula are given, respectively, by (see $[1,2]$ )

$$
\begin{gathered}
\widetilde{\nabla}_{X} Y=\nabla_{X} Y+h(X, Y), \\
\widetilde{\nabla}_{X} \xi=-A_{\xi} X+D_{X} \xi,
\end{gathered}
$$

for vector fields $X, Y$ tangent to $M$ and $\xi$ normal to $M$, where $h, A$, and $D$ are the second fundamental form, the shape operator, and the normal connection. It is well known that the second fundamental form $h$ and the shape operator $A$ are related by

$$
\langle h(X, Y), \xi\rangle=\left\langle A_{\xi} X, Y\right\rangle,
$$

for $X, Y$ tangent to $M$ and $\xi$ normal to $M$.

A vector $v$ is called spacelike (timelike) if $\langle v, v\rangle>0$ or $\langle v, v\rangle=0(\langle v, v\rangle\langle 0)$. A vector $v$ is called lightlike if it is nonzero and it satisfies $\langle v, v\rangle=0$.

We define the light cone $\mathscr{L} \mathscr{C} \subset \mathbb{C}_{1}^{2}$ by $\left\{w \in \mathbb{C}_{1}^{2} \mid\langle w, w\rangle=\right.$ $0\}$. A curve $w(t)$ is called null if $w^{\prime}$ is lightlike for any $t$.

For each normal vector $\xi$ of $M$ at $x \in M$, the shape operator $A_{\xi}$ is a symmetric endomorphism of the tangent space $T_{x} M$. The mean curvature vector is defined by

$$
H=\frac{1}{2} \text { trace } h .
$$

A Lorentzian surface $M$ in $\widetilde{M}_{1}^{2}$ is called minimal if its mean curvature vector $H$ vanishes at each point on $M$. And a Lorentzian surface $M$ in $\widetilde{M}_{1}^{2}$ is called marginally trapped (or quasiminimal) if its mean curvature vector is lightlike at each point on $M$.

For a Lorentzian surface $M$ in a Lorentzian complex space form $\widetilde{M}_{1}^{2}$, the Gauss and Codazzi and Ricci equations are given, respectively, by

$$
\begin{aligned}
&\langle R(X, Y) Z, W\rangle=\langle\widetilde{R}(X, Y) Z, W\rangle \\
&+\langle h(Y, Z), h(X, W)\rangle \\
&-\langle h(X, Z), h(Y, W)\rangle, \\
&(\widetilde{R}(X, Y) Z)^{\perp}=(\bar{\nabla} h)(X, Y, Z)-(\bar{\nabla} h)(Y, X, Z), \\
&\left\langle R^{D}(X, Y) \xi, \eta\right\rangle=\langle\bar{R}(X, Y) \xi, \eta\rangle \\
&+\left\langle\left[A_{\xi}, A_{\eta}\right] X, Y\right\rangle,
\end{aligned}
$$

where $X, Y, Z$, and $W$ are vectors tangent to $M$ and $\bar{\nabla} h$ is defined by

$$
(\bar{\nabla} h)(X, Y, Z)=D_{X} h(Y, Z)-h\left(\nabla_{X} Y, Z\right)-h\left(Y, \nabla_{X} Z\right) .
$$


2.2. Bitension Field. For smooth maps $\phi:\left(M^{n}, g\right) \rightarrow\left(\widetilde{M}^{m}\right.$, $\langle\rangle$,$) , the tension field \tau(\phi)$ is a section of the vector bundle $\phi^{*} T \widetilde{M}$ defined by

$$
\tau(\phi)=\operatorname{trace} \nabla \mathrm{d} \phi=\sum_{i=1}^{n}\left\langle e_{i}, e_{i}\right\rangle\left\{\nabla_{e_{i}}^{\phi} d \phi\left(e_{i}\right)-d \phi\left(\nabla_{e_{i}} e_{i}\right)\right\},
$$

where $\nabla^{\phi}$ is the induced connection by $\phi$ on the bundle $\phi^{*} T \widetilde{M}$, which is the pullback of $\widetilde{\nabla}$. If $\phi$ is an isometric immersion, then $\tau(\phi)$ and the mean curvature vector field $H$ of $M$ are related by

$$
\tau(\phi)=n H .
$$

If $\tau(\phi)=0$ at each point on $M$, then $\phi$ is called a harmonic map. The harmonic maps between two Riemannian manifolds are critical points of the energy functional

$$
E(\phi)=\frac{1}{2} \int_{M}|d \phi|^{2} v_{g}
$$

for smooth maps $\phi:\left(M^{n}, g\right) \rightarrow\left(\widetilde{M}^{m},\langle\rangle,\right)$.

The bitension field is defined by

$$
\begin{aligned}
\tau_{2}(\phi)=\sum_{i=1}^{n}\left\langle e_{i}, e_{i}\right\rangle\{ & \left(\nabla_{e_{i}}^{\phi} \nabla_{e_{i}}^{\phi}-\nabla_{\nabla_{e_{i}} e_{i}}^{\phi}\right) \tau \\
& \left.+R^{N}\left(\tau, d \phi\left(e_{i}\right)\right) d \phi\left(e_{i}\right)\right\},
\end{aligned}
$$

where $R^{\widetilde{M}}$ is the curvature tensor of $\widetilde{M}$.

If $\phi$ is an isometric immersion and $\widetilde{M}$ is the complex space form $\widetilde{M}_{i}^{n}(4 c)$, it follows from (1), (14), and (16) that

$$
\tau_{2}(\phi)=-n \Delta H+5 n c H,
$$

where $\Delta=-\sum_{i=1}^{n}\left\langle e_{i}, e_{i}\right\rangle\left(\widetilde{\nabla}_{e_{i}} \widetilde{\nabla}_{e_{i}}-\widetilde{\nabla}_{\nabla_{e_{i}} e_{i}}\right)$.

A smooth map $\phi$ is called biharmonic if $\tau_{2}(\phi)=0$ at each point on $M$. It is easy to see that harmonic maps are always biharmonic.

Biharmonic maps $\phi:\left(M^{n}, g\right) \rightarrow\left(\widetilde{M}^{m},\langle\rangle,\right)$ between Riemannian manifolds are critical points of the bienergy functional

$$
E_{2}(\phi)=\frac{1}{2} \int_{M}|\tau(\phi)|^{2} v_{g} .
$$

Sasahara proposed the notion of quasi-biharmonic submanifolds as follows.

Definition 1. A pseudo-Riemannian submanifold isometrically immersed in a pseudo-Riemannian manifold by $\phi$ is called quasi-biharmonic if $\tau_{2}$ is lightlike at each point on the submanifold.

\section{Basic Results on Lorentzian Slant Surfaces}

Let $M$ be a Lorentzian surface in a Lorentzian Kähler surface $\left(\widetilde{M}_{1}^{2}, g, J\right)$. For each tangent vector $X$ of $M$, we put

$$
J X=P X+F X,
$$

where $P X$ and $F X$ are the tangential and the normal components of $J X$.
On the Lorentzian surface $M$ there exists a pseudoorthonormal local frame $\left\{e_{1}, e_{2}\right\}$ such that

$$
\left\langle e_{1}, e_{1}\right\rangle=\left\langle e_{2}, e_{2}\right\rangle=0, \quad\left\langle e_{1}, e_{2}\right\rangle=-1 .
$$

It follows from (19), (20), and $\langle J X, J Y\rangle=\langle X, Y\rangle$ that

$$
P e_{1}=(\sinh \theta) e_{1}, \quad P e_{2}=-(\sinh \theta) e_{2},
$$

for some function $\theta$. This function $\theta$ is called the Wirtinger angle of $M$.

When the Wirtinger angle $\theta$ is constant on $M$, the Lorentzian surface $M$ is called a slant surface (cf. [2, 3]). In this case, $\theta$ is called the slant angle; the slant surface is then called $\theta$-slant.

A $\theta$-slant surface is called Lagrangian if $\theta=0$ and proper slant if $\theta \neq 0$.

If we put

$$
e_{3}=(\operatorname{sech} \theta) F e_{1}, \quad e_{4}=(\operatorname{sech} \theta) F e_{2},
$$

then we find from (19)-(22) that

$$
\begin{array}{r}
J e_{1}=\sinh \theta e_{1}+\cosh \theta e_{3}, \quad J e_{2}=-\sinh \theta e_{2}+\cosh \theta e_{4}, \\
J e_{3}=-\cosh \theta e_{1}-\sinh \theta e_{3}, \quad J e_{4}=-\cosh \theta e_{2}+\sinh \theta e_{4}, \\
\left\langle e_{3}, e_{3}\right\rangle=\left\langle e_{4}, e_{4}\right\rangle=0, \quad\left\langle e_{3}, e_{4}\right\rangle=-1 .
\end{array}
$$

We call such a frame $\left\{e_{1}, e_{2}, e_{3}, e_{4}\right\}$ an adapted pseudoorthonormal frame for the Lorentzian surface $M$ in $\widetilde{M}_{1}^{2}$.

Lemma 2. If $M$ is a slant surface in a Lorentzian Kähler surface $\widetilde{M}_{1}^{2}$, then with respect to an adapted pseudoorthonormal frame one has

$$
\begin{array}{ll}
\nabla_{X} e_{1}=\omega(X) e_{1}, & \nabla_{X} e_{2}=-\omega(X) e_{2}, \\
D_{X} e_{3}=\Phi(X) e_{3}, & D_{X} e_{4}=-\Phi(X) e_{4},
\end{array}
$$

for some 1-forms $\omega$, $\Phi$ on $M$.

For a Lorentzian surface $M$ in $\widetilde{M}_{1}^{2}$, we put

$$
h\left(e_{i}, e_{j}\right)=h_{i j}^{3} e_{3}+h_{i j}^{4} e_{4},
$$

where $\left\{e_{1}, e_{2}, e_{3}, e_{4}\right\}$ is an adapted pseudoorthonormal frame and $h$ is the second fundamental form of $M$.

Lemma 3 (see [3]). If $M$ is a $\theta$-slant surface in a Lorentzian Kähler surface $\widetilde{M}_{1}^{2}$, then with respect to an adapted pseudoorthonormal frame one has

$$
\begin{gathered}
\omega_{j}-\Phi_{j}=2 h_{1 j}^{3} \tanh \theta, \\
A_{F X} Y=A_{F Y} X, \\
A_{e_{3}} e_{j}=h_{1 j}^{3} e_{1}+h_{1 j}^{4} e_{2}, \quad A_{e_{4}} e_{j}=h_{j 2}^{3} e_{1}+h_{j 2}^{4} e_{2},
\end{gathered}
$$

for any $X, Y \in T M$ and $j=1,2$, where $\omega_{j}=\omega\left(e_{j}\right)$ and $\Phi_{j}=$ $\Phi\left(e_{j}\right)$. 
For Lorentzian slant surfaces in $\widetilde{M}_{1}^{2}(4 c)$, the author with Hou has proved the following interesting result.

Theorem 4 (see [17]). Every slant surface in a nonflat Lorentzian complex space form $\widetilde{M}_{1}^{2}(4 c)$ must be Lagrangian.

According to Theorem 4, we need only to consider the slant surfaces in Lorentzian complex plane $\mathbb{C}_{1}^{2}$ because the case of Lagrangian marginally trapped surfaces has been considered in $[16,18]$.

\section{The Bitension Field of Marginally Trapped Slant Surfaces}

Let $M$ be a $\theta$-slant marginally trapped surface in a Lorentzian complex plane $\mathbb{C}_{1}^{2}$. There is a pseudoorthonormal local frame field $\left\{\widehat{e}_{1}, \widehat{e}_{2}\right\}$ such that

$$
\begin{gathered}
\left\langle\widehat{e}_{1}, \widehat{e}_{1}\right\rangle=\left\langle\widehat{e}_{2}, \widehat{e}_{2}\right\rangle=0, \quad\left\langle\widehat{e}_{1}, \widehat{e}_{2}\right\rangle=-1, \\
H=-h\left(\widehat{e}_{1}, \widehat{e}_{2}\right) .
\end{gathered}
$$

Since the mean curvature vector $H$ is lightlike at each point, we put $h\left(\widehat{e}_{1}, \widehat{e}_{2}\right)=\alpha F \widehat{e}_{1}$ for some nonzero real-valued function $\alpha$. By putting $e_{1}=\alpha \widehat{e}_{1}, e_{2}=\alpha^{-1} \widehat{e}_{2}$, we have

$$
\begin{gathered}
\left\langle e_{1}, e_{1}\right\rangle=\left\langle e_{2}, e_{2}\right\rangle=0, \quad\left\langle e_{1}, e_{2}\right\rangle=-1, \\
h\left(e_{1}, e_{2}\right)=F e_{1} .
\end{gathered}
$$

By applying (20) and the total symmetry of $\langle h(X, Y), F Z\rangle$, we obtain

$$
\begin{gathered}
h\left(e_{1}, e_{1}\right)=\lambda F e_{2}, \quad h\left(e_{1}, e_{2}\right)=F e_{1}, \\
h\left(e_{2}, e_{2}\right)=\gamma F e_{1}+F e_{2},
\end{gathered}
$$

for two smooth functions $\lambda, \gamma$. It follows from (9), (22), (25), and (33) that

$$
A_{\mathrm{Fe}_{1}}=\cosh ^{2} \theta\left(\begin{array}{ll}
0 & 1 \\
\lambda & 0
\end{array}\right), \quad A_{\mathrm{Fe}_{2}}=\cosh ^{2} \theta\left(\begin{array}{ll}
1 & \gamma \\
0 & 1
\end{array}\right) \text {. }
$$

By Lemma 2, (33), and differentiating the second fundamental form covariantly, we get

$$
\begin{gathered}
\left(\bar{\nabla}_{e_{2}} h\right)\left(e_{1}, e_{1}\right)=e_{2}(\lambda) F e_{2}-\lambda \Phi_{2} F e_{2}-2 \omega_{2} \lambda F e_{2}, \\
\left(\bar{\nabla}_{e_{1}} h\right)\left(e_{1}, e_{2}\right)=\Phi_{1} F e_{1}, \\
\left(\bar{\nabla}_{e_{2}} h\right)\left(e_{1}, e_{2}\right)=\Phi_{2} F e_{1}, \\
\left(\bar{\nabla}_{e_{1}} h\right)\left(e_{2}, e_{2}\right)=e_{1}(\gamma) F e_{1}+\gamma \Phi_{1} F e_{1}-\Phi_{1} F e_{2} \\
+2 \omega_{1} \gamma F e_{1}+2 \omega_{1} F e_{2} .
\end{gathered}
$$

By the Codazzi equation, comparing coefficients gives

$$
\begin{gathered}
\omega_{1}=\Phi_{1}=0, \quad e_{2}(\lambda)-\lambda \Phi_{2}-2 \omega_{2} \lambda=0, \\
e_{1}(\gamma)-\Phi_{2}=0 .
\end{gathered}
$$

On the other hand, from Lemma 3 and (33) we have

$$
\omega_{2}-\Phi_{2}=2 \sinh \theta
$$

Consequently, (36) becomes

$$
\begin{gathered}
\omega_{1}=0, \quad e_{2}(\lambda)=3 \omega_{2} \lambda-2 \sinh \theta \lambda, \\
e_{1}(\gamma)=\omega_{2}-2 \sinh \theta .
\end{gathered}
$$

In order to express the bitension field of marginally trapped slant surfaces in $\mathbb{C}_{1}^{2}$ with respect to a pseudoorthonormal frame (31), we need the following formula (cf. $[1,18])$ :

$$
\begin{aligned}
\Delta H= & \Delta^{D} H-h\left(e_{1}, A_{H} e_{2}\right)-h\left(e_{2}, A_{H} e_{1}\right) \\
& -A_{D_{e_{1}} H_{2}}-A_{D_{e_{2}} H} e_{1}-\left(\nabla_{e_{1}} A_{H}\right) e_{2}-\left(\nabla_{e_{2}} A_{H}\right) e_{1},
\end{aligned}
$$

where $\Delta$ and $\Delta^{D}$ are, respectively, given by

$$
\begin{gathered}
\Delta=\widetilde{\nabla}_{e_{1}} \widetilde{\nabla}_{e_{2}}+\widetilde{\nabla}_{e_{2}} \widetilde{\nabla}_{e_{1}}-\widetilde{\nabla}_{\nabla_{e_{1}} e_{2}}-\widetilde{\nabla}_{\nabla_{e_{2}} e_{1}}, \\
\Delta^{D}=D_{e_{1}} D_{e_{2}}+D_{e_{2}} D_{e_{1}}-D_{\nabla_{e_{1}} e_{2}}-D_{\nabla_{e_{2}} e_{1}} .
\end{gathered}
$$

Lemma 5. Let $M$ be a marginally trapped slant surface in $\mathbb{C}_{1}^{2}$. Then, the Gauss curvatures $K$ and $\Delta^{D} H$ are related by

$$
\Delta^{D} H=-K H .
$$

Proof. It follows from (33) that the mean curvature vector $H$ is given by $H=-F e_{1}$. By (22), (26), (27), (36), and (37), we have

$$
\begin{gathered}
D_{e_{1}} D_{e_{2}} H=-e_{1}\left(\Phi_{2}\right) F e_{1}=-e_{1}\left(\omega_{2}\right) F e_{1}, \\
D_{e_{2}} D_{e_{1}} H=D_{\nabla_{e_{1}} e_{2}} H=D_{\nabla_{e_{2}} e_{1}} H=0 .
\end{gathered}
$$

Consequently, we obtain

$$
\Delta^{D} H=-e_{1}\left(\omega_{2}\right) F e_{1} .
$$

Recall the definition of the Gauss curvature $K=$ $-\left\langle R\left(e_{1}, e_{2}\right) e_{2}, e_{1}\right\rangle$. It follows from Lemma 2 and $\omega_{1}=0$ that

$$
K=-e_{1}\left(\omega_{2}\right) \text {, }
$$

which completes the proof of Lemma 5.

Remark 6. For marginally trapped Lagrangian surfaces immersed into Lorentzian complex space forms, Sasahara [15] has proved the formula $\Delta^{D} H=-K H$. Hence, we know from Lemma 5 that the formula $\Delta^{D} H=-K H$ also holds for slant surfaces in Lorentzian complex space forms.

Lemma 7. Let $M$ be a marginally trapped slant surface in $\mathbb{C}_{1}^{2}$. Then, the normal part of $\Delta H$ is expressed as

$$
(\Delta H)^{\perp}=2 \cosh ^{2} \theta \lambda\left(\gamma F e_{1}+F e_{2}\right) .
$$


Proof. On one hand, it follows from (34) that

$$
A_{H} e_{2}=-\cosh ^{2} \theta e_{1}, \quad A_{H} e_{1}=-\cosh ^{2} \theta \lambda e_{2} .
$$

Combining (46) with (33) gives

$$
h\left(e_{1}, A_{H} e_{2}\right)+h\left(e_{2}, A_{H} e_{1}\right)=-\cosh ^{2} \theta\left(\lambda \gamma F e_{1}+2 \lambda F e_{2}\right) .
$$

On the other hand, it follows from (33) and the Gauss equation that the Gauss curvature is given by

$$
K=\cosh ^{2} \theta \lambda \gamma
$$

By Lemma 5, we have $\Delta^{D} H=\cosh ^{2} \theta \lambda \gamma F e_{1}$. Combining these with the first section of (39) completes the proof.

Lemma 8. Let $M$ be a marginally trapped slant surface in $\mathbb{C}_{1}^{2}$. Then, the tangential part of $\Delta H$ is expressed as

$$
(\Delta H)^{\top}=\cosh ^{2} \theta\left(e_{2}(\lambda)-\lambda \omega_{2}-2 \lambda \sinh \theta\right) e_{2} .
$$

Proof. By (27), (36), and (46), we obtain

$$
-A_{D_{e_{1}} H} e_{2}-A_{D_{e_{2}}{ }} e_{1}=-\Phi_{2} A_{H} e_{1}=\cosh ^{2} \theta \Phi_{2} \lambda e_{2} .
$$

It follows from (26), (38), and (46) that

$$
\begin{aligned}
\left(\nabla_{e_{1}} A_{H}\right) e_{2} & =\nabla_{e_{1}}\left(A_{H} e_{2}\right)-A_{H}\left(\nabla_{e_{1}} e_{2}\right) \\
& =-2 \cosh ^{2} \theta \omega_{1} e_{1}=0 \\
\left(\nabla_{e_{2}} A_{H}\right) e_{1} & =\nabla_{e_{2}}\left(A_{H} e_{1}\right)-A_{H}\left(\nabla_{e_{2}} e_{1}\right) \\
& =-\cosh ^{2} \theta\left(e_{2}(\lambda)-2 \lambda \omega_{2}\right) e_{2} .
\end{aligned}
$$

Combing (50)-(51) with (37) gives the conclusion.

Hence, by (17) and Lemmas 7 and 8, we get the expression of the bitension field of marginally trapped slant surfaces in Lorentzian complex plane $\mathbb{C}_{1}^{2}$.

Lemma 9. Let $\phi: M \rightarrow \mathbb{C}_{1}^{2}$ be a marginally trapped slant immersed in $\mathbb{C}_{1}^{2}$. Then the bitension field is given by

$$
\begin{aligned}
\tau_{2}(\phi)= & -2 \cosh ^{2} \theta\left(e_{2}(\lambda)-\lambda \omega_{2}-2 \lambda \sinh \theta\right) e_{2} \\
& -4 \cosh ^{2} \theta \lambda\left(\gamma F e_{1}+F e_{2}\right)
\end{aligned}
$$

\section{Classification Results}

From now on, let us consider the biharmonic and quasibiharmonic Marginally trapped slant surfaces in Lorentzian complex plane $\mathbb{C}_{1}^{2}$.

By the definition of biharmonic surfaces, we can conclude the following from Lemma 9.

Lemma 10. Let $\phi: M \rightarrow \mathbb{C}_{1}^{2}$ be a marginally trapped slant immersed in $\mathbb{C}_{1}^{2}$. Then the immersion is biharmonic if and only if the function $\lambda$ satisfies $\lambda=0$.
Similarly, by the definition of quasi-biharmonic submanifolds, the bitension field $\tau_{2}(\phi)$ is lightlike. Therefore, we also have the following.

Lemma 11. Let $\phi: M \rightarrow \mathbb{C}_{1}^{2}$ be a marginally trapped slant immersed in $\mathbb{C}_{1}^{2}$. Then the immersion is quasi-biharmonic if and only if the functions $\lambda$ and $\gamma$ satisfy $\lambda \neq 0$ and $\gamma=0$.

Since the Gauss curvature $K$ is given by (48), we deduce from Lemmas 10 and 11 that $K=0$ in both cases. Moreover, (44) implies that $e_{1}\left(\omega_{2}\right)=0$.

We deduce from $(26)$ and (38) that $\left[e_{1}, e_{2}\right]=-\omega_{2} e_{1}$. There is a nonzero smooth function $\beta$ satisfying

$$
e_{2}(\beta)+\beta \omega_{2}=0
$$

such that $\left[\beta e_{1}, e_{2}\right]=0$. Thus, there exist local coordinates $(x, y)$ on $M$ such that $\partial / \partial x=\beta e_{1}$ and $\partial / \partial y=e_{2}$. Then the metric tensor of $M$ is given by

$$
g=-\beta(d x \otimes d y+d y \otimes d x),
$$

and the Levi-Civita connection of $g$ satisfies

$$
\nabla_{\partial / \partial x} \frac{\partial}{\partial x}=\frac{\beta_{x}}{\beta} \frac{\partial}{\partial x}, \quad \nabla_{\partial / \partial x} \frac{\partial}{\partial y}=0, \quad \nabla_{\partial / \partial y} \frac{\partial}{\partial y}=\frac{\beta_{y}}{\beta} \frac{\partial}{\partial y} .
$$

Moreover, it follows from (33) and (54) that

$$
\begin{gathered}
h\left(\frac{\partial}{\partial x}, \frac{\partial}{\partial x}\right)=\lambda \beta^{2} F \frac{\partial}{\partial y}, \quad h\left(\frac{\partial}{\partial x}, \frac{\partial}{\partial y}\right)=F \frac{\partial}{\partial x} \\
h\left(\frac{\partial}{\partial y}, \frac{\partial}{\partial y}\right)=\gamma \beta^{-1} F \frac{\partial}{\partial x}+F \frac{\partial}{\partial y} .
\end{gathered}
$$

Since $e_{1}\left(\omega_{2}\right)=0$, it follows that $\omega_{2}$ is a function depending only on $y$; that is, $\omega_{2}=\omega_{2}(y)$. Using local coordinates, (53) becomes

$$
\beta_{y}+\beta \omega_{2}(y)=0
$$

Solving (57) gives

$$
\beta=e^{-\int \omega_{2}(y) d y}
$$

Without loss of generality, we may assume that $\beta$ is only depending on variable $y$.

By applying (23), (55), (56), (58), and Gauss formula (7), we have the following PDE system:

$$
\begin{gathered}
L_{x x}=\lambda \beta^{2}(i+\sinh \theta) L_{y}, \\
L_{x y}=(i-\sinh \theta) L_{x}, \\
L_{y y}=\beta^{-1} \gamma(i-\sinh \theta) L_{x}+\left(\frac{\beta y}{\beta}+i+\sinh \theta\right) L_{y} .
\end{gathered}
$$

By solving (60), we obtain that

$$
L(x, y)=f(x) e^{(i-\sinh \theta) y}+z(y)
$$

for some vector-valued functions $f(x)$ and $z(y)$ in $\mathbb{C}_{1}^{2}$. 
Theorem 12. Up to rigid motions of $\mathbb{C}_{1}^{2}$, every biharmonic marginally trapped $\theta$-slant surface in $\mathbb{C}_{1}^{2}$ is given by a flat slant surface defined by

$$
L(x, y)=c_{1} x e^{(i-\sinh \theta) y}+w(y),
$$

where $c_{1}$ is lightlike vector and $w(y)$ is a null curve in $\mathbb{C}_{1}^{2}$ satisfying

$$
\begin{aligned}
& \left\langle c_{1}(i-\sinh \theta) e^{(i-\sinh \theta) y}, w^{\prime}(y)\right\rangle=0, \\
& \left\langle c_{1} e^{(i-\sinh \theta) y}, w^{\prime}(y)\right\rangle=-\beta(y)
\end{aligned}
$$

for some nonzero real-valued function $\beta(y)$.

Proof. Let $M$ be a biharmonic marginally trapped $\theta$-slant surface in $\mathbb{C}_{1}^{2}$. According to Lemma 10, we have $\lambda=0$. Substituting (62) into (59), we have

$$
f^{\prime \prime}(x)=0,
$$

which yields $f(x)=c_{1} x+c_{2}$ for two constant vectors $c_{1}$ and $c_{2}$ in $\mathbb{C}_{1}^{2}$. Hence, the immersion becomes

$$
L(x, y)=c_{1} x e^{(i-\sinh \theta) y}+w(y) .
$$

Note that $w(y)=c_{2} e^{(i-\sinh \theta) y}+z(y)$ here. Moreover, (66) yields

$$
\begin{gathered}
L_{x}=c_{1} e^{(i-\sinh \theta) y}, \\
L_{y}=c_{1}(i-\sinh \theta) x e^{(i-\sinh \theta) y}+w^{\prime}(y) .
\end{gathered}
$$

It follows from (54) and (67) that

$$
\begin{aligned}
\left\langle c_{1}, c_{1}\right\rangle & =\left\langle w^{\prime}(y), w^{\prime}(y)\right\rangle \\
& =\left\langle c_{1}(i-\sinh \theta) e^{(i-\sinh \theta) y}, w^{\prime}(y)\right\rangle=0, \\
& \left\langle c_{1} e^{(i-\sinh \theta) y}, w^{\prime}(y)\right\rangle=-\beta(y) .
\end{aligned}
$$

This completes the proof of Theorem 12 .

Theorem 13. Up to rigid motions of $\mathbb{C}_{1}^{2}$, every quasibiharmonic marginally trapped $\theta$-slant surface in $\mathbb{C}_{1}^{2}$ is given by a flat slant surface defined by

$$
L(x, y)=p(x) e^{(i-\sinh \theta) y},
$$

where $p(x)$ is a null curve lying in the light cone $\mathscr{L} \mathscr{C}$ satisfying

$$
\begin{gathered}
\langle p(x), p(x)\rangle=\left\langle p^{\prime}(x), p^{\prime}(x)\right\rangle=0, \\
\left\langle p(x), i p^{\prime}(x)\right\rangle=1 .
\end{gathered}
$$

Proof. Let $M$ be a quasi-biharmonic marginally trapped $\theta$ slant surface in $\mathbb{C}_{1}^{2}$. According to Lemma 11, we have $\gamma=0$ and $\lambda \neq 0$. In this case, the second and third equations of (38) become

$$
\omega_{2}=2 \sinh \theta, \quad \lambda_{y}=4 \sinh \theta \lambda .
$$

Solving $\lambda$, we get

$$
\lambda=c_{1}(x) e^{4 \sinh \theta y} .
$$

By applying (71), (58) yields

$$
\beta=a e^{-2 \sinh \theta y}
$$

for some nonzero constant number $a$. Consequently, with the previous information, the PDE system (59)-(61) becomes

$$
\begin{gathered}
L_{x x}=a c_{1}(x) L_{y}, \\
L_{x y}=(i-\sinh \theta) L_{x}, \\
L_{y y}=(i-\sinh \theta) L_{y} .
\end{gathered}
$$

Substituting (62) into (76), we have

$$
z^{\prime \prime}(y)=(i-\sinh \theta) z^{\prime}(y),
$$

which yields

$$
z(y)=c_{2} e^{(i-\sinh \theta) y}+c_{3}
$$

for two constant vectors $c_{2}$ and $c_{3}$ in $\mathbb{C}_{1}^{2}$. We denote $p(x)$ by $p(x)=f(x)+c_{2}$. Hence, up to rigid motions of $\mathbb{C}_{1}^{2}$ the immersion becomes

$$
L(x, y)=p(x) e^{(i-\sinh \theta) y} .
$$

Substituting (79) into (74), we obtain

$$
p^{\prime \prime}(x)=-a \cosh ^{2} \theta c_{1}(x) p(x) .
$$

Moreover, (79) gives

$$
L_{x}=p^{\prime}(x) e^{(i-\sinh \theta) y}, \quad L_{y}=(i-\sinh \theta) p(x) e^{(i-\sinh \theta) y} .
$$

It follows from (54), (73), and (81) that

$$
\begin{gathered}
\langle p(x), p(x)\rangle=\left\langle p^{\prime}(x), p^{\prime}(x)\right\rangle=0, \\
\left\langle p^{\prime}(x),(i-\sinh \theta) p(x)\right\rangle=-a .
\end{gathered}
$$

In view of (73), we can assume $a=1$. Hence, we get the conclusion.

Remark 14. According to Theorem 4, combining Theorems 12 and 13 with Sasahara's results in $[16,18]$, we finish the complete classifications of biharmonic marginally trapped slant surfaces and quasi-biharmonic marginally trapped slant surfaces in Lorentzian complex forms, respectively.

\section{Acknowledgments}

This work is supported by the Natural Science Foundation of China (no. 71271045), Program for Liaoning Excellent Talents in University (no. LJQ2012099), and General Project for Scientific Research of Liaoning Educational Committee (no. W2012186). 


\section{References}

[1] B.-Y. Chen, Pseudo-Riemannian Geometry, $\delta$-Invariants and Applications, World Scientific Publishing, Hackensack, NJ, USA, 2011.

[2] B.-Y. Chen, Geometry of Slant Submanifolds, Katholieke Universiteit Leuven, Leuven, Belgium, 1990.

[3] B. Y. Chen and I. Mihai, "Classification of quasi-minimal slant surfaces in Lorentzian complex space forms," Acta Mathematica Hungarica, vol. 122, no. 4, pp. 307-328, 2009.

[4] K. Kenmotsu and D. Zhou, "The classification of the surfaces with parallel mean curvature vector in two-dimensional complex space forms," American Journal of Mathematics, vol. 122, no. 2, pp. 295-317, 2000.

[5] K. Arslan, A. Carriazo, B.-Y. Chen, and C. Murathan, "On slant submanifolds of neutral Kaehler manifolds," Taiwanese Journal of Mathematics, vol. 14, no. 2, pp. 561-584, 2010.

[6] B.-Y. Chen, "Minimal flat Lorentzian surfaces in Lorentzian complex space forms," Publicationes Mathematicae Debrecen, vol. 73, no. 1-2, pp. 233-248, 2008.

[7] B.-Y. Chen, "Dependence of the Gauss-Codazzi equations and the Ricci equation of Lorentz surfaces," Publicationes Mathematicae Debrecen, vol. 74, no. 3-4, pp. 341-349, 2009.

[8] B.-Y. Chen, "Nonlinear Klein-Gordon equations and Lorentzian minimal surfaces in Lorentzian complex space forms," Taiwanese Journal of Mathematics, vol. 13, no. 1, pp. 1-24, 2009.

[9] B.-Y. Chen and F. Dillen, "Classification of marginally trapped Lagrangian surfaces in Lorentzian complex space forms," Journal of Mathematical Physics, vol. 48, no. 1, Article ID 013509, 23 pages, 2007.

[10] B.-Y. Chen and S. Ishikawa, "Biharmonic surfaces in pseudoEuclidean spaces," Memoirs of the Faculty of Science. Kyushu University A, vol. 45, no. 2, pp. 323-347, 1991.

[11] B.-Y. Chen and S. Ishikawa, "Biharmonic pseudo-Riemannian submanifolds in pseudo-Euclidean spaces," Kyushu Journal of Mathematics, vol. 52, no. 1, pp. 167-185, 1998.

[12] B.-Y. Chen, "Black holes, marginally trapped surfaces and quasiminimal surfaces," Tamkang Journal of Mathematics, vol. 40, no. 4, pp. 313-341, 2009.

[13] L. Vrancken, "Minimal Lagrangian submanifolds with constant sectional curvature in indefinite complex space forms," Proceedings of the American Mathematical Society, vol. 130, no. 5, pp. 1459-1466, 2002.

[14] S. Montaldo and C. Oniciuc, "A short survey on biharmonic maps between Riemannian manifolds," Revista de la Unión Matemática Argentina, vol. 47, no. 2, pp. 1-22, 2006.

[15] T. Sasahara, "Quasi-minimal Lagrangian surfaces whose mean curvature vectors are eigenvectors," Demonstratio Mathematica, vol. 38, no. 1, pp. 185-196, 2005.

[16] T. Sasahara, "Quasi-biharmonic Lagrangian surfaces in Lorentzian complex space forms," Annali di Matematica Pura ed Applicata, vol. 192, no. 2, pp. 191-201, 2013.

[17] Y. Fu and Z. H. Hou, "Classification of pseudo-umbilical slant surfaces in Lorentzian complex space forms," Taiwanese Journal of Mathematics, vol. 15, no. 5, pp. 1919-1938, 2011.

[18] T. Sasahara, "Biharmonic Lagrangian surfaces of constant mean curvature in complex space forms," Glasgow Mathematical Journal, vol. 49, no. 3, pp. 497-507, 2007. 


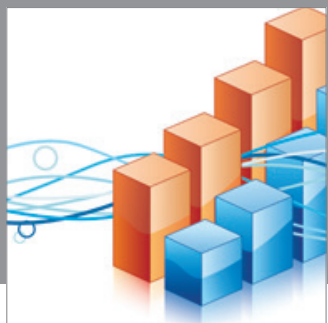

Advances in

Operations Research

mansans

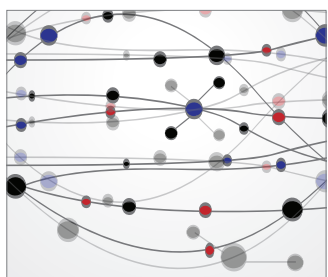

The Scientific World Journal
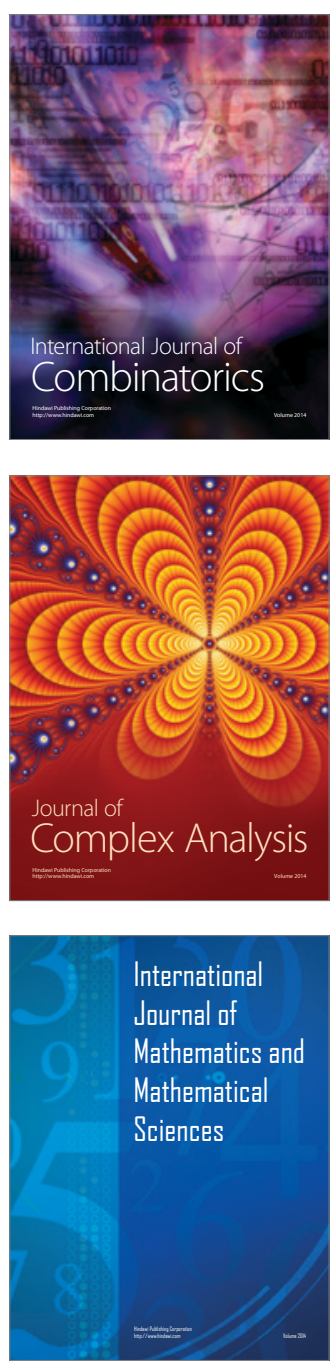
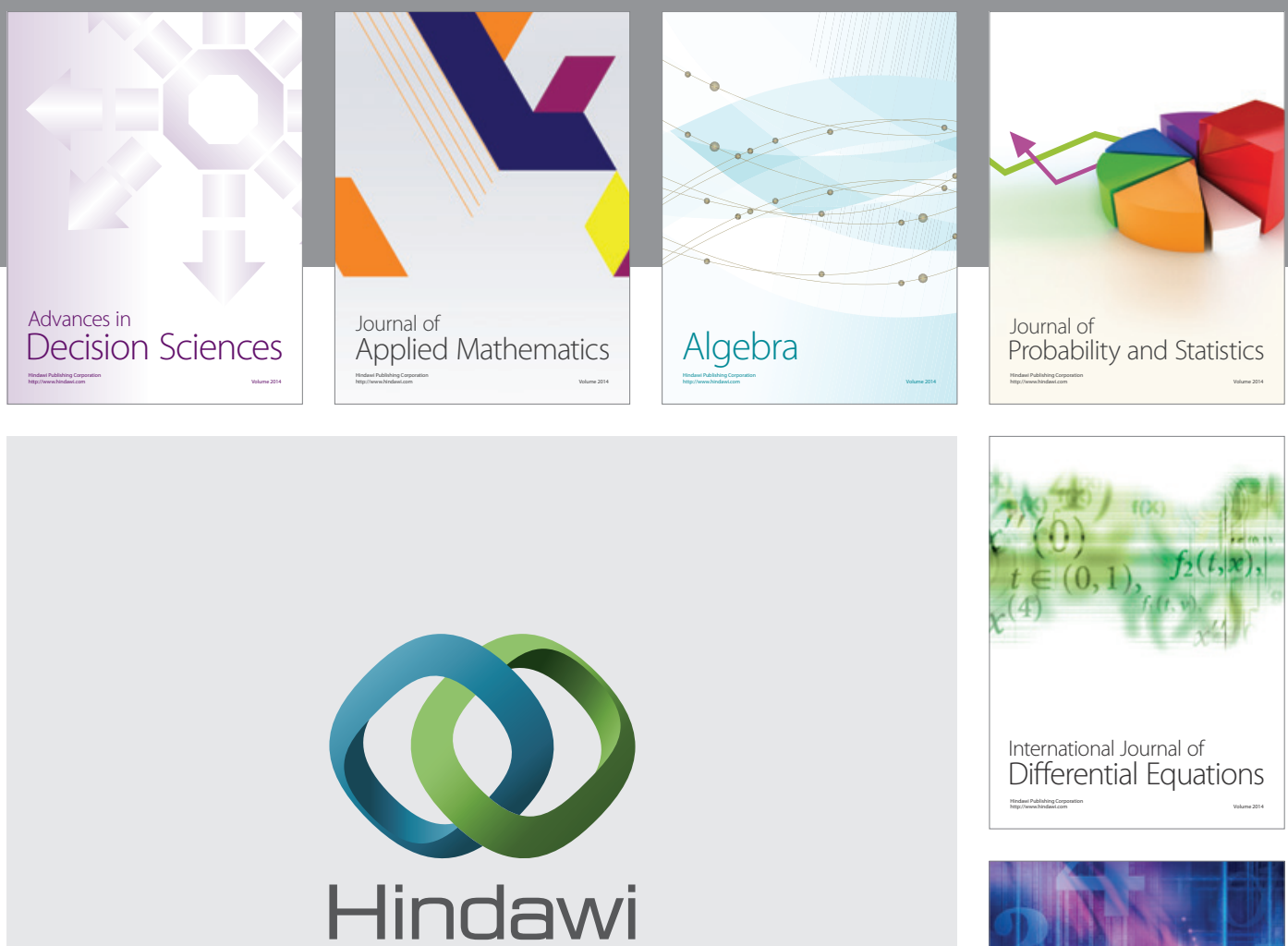

Submit your manuscripts at http://www.hindawi.com
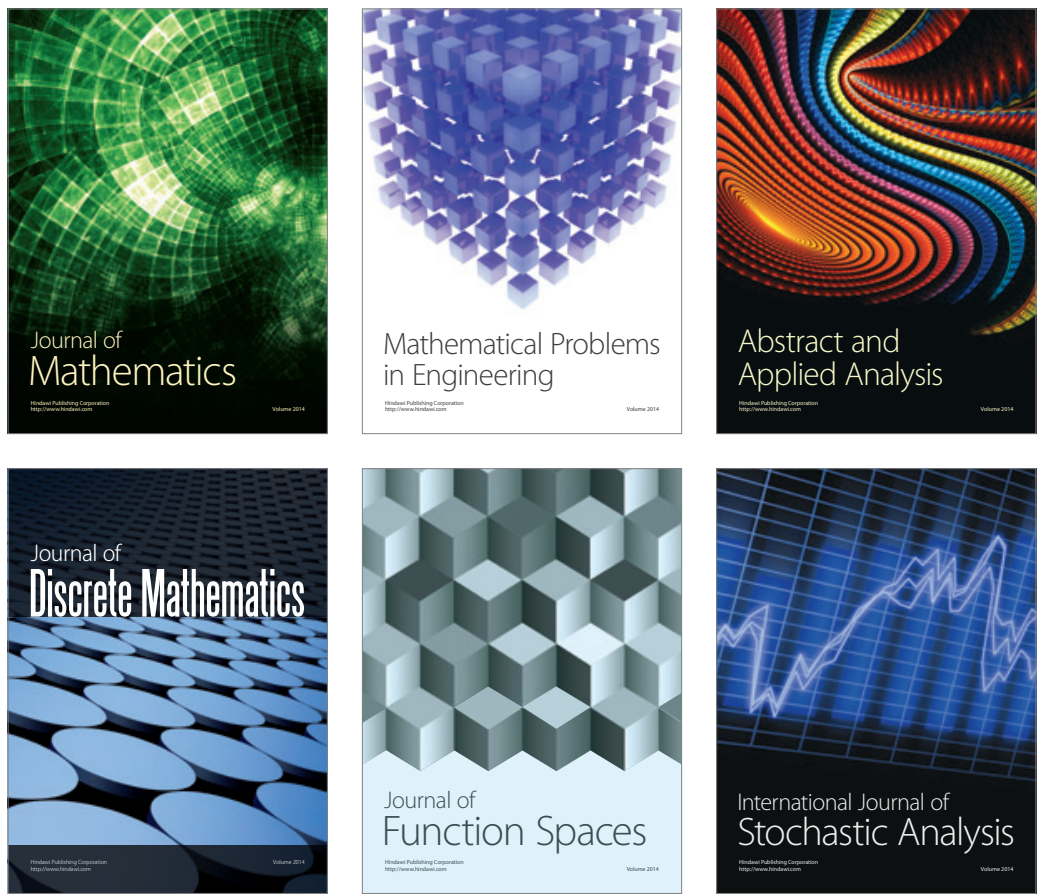

Journal of

Function Spaces

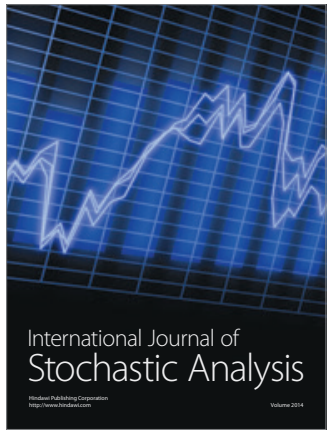

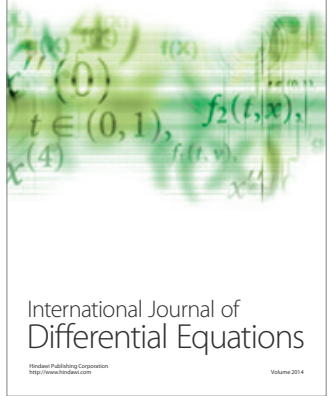
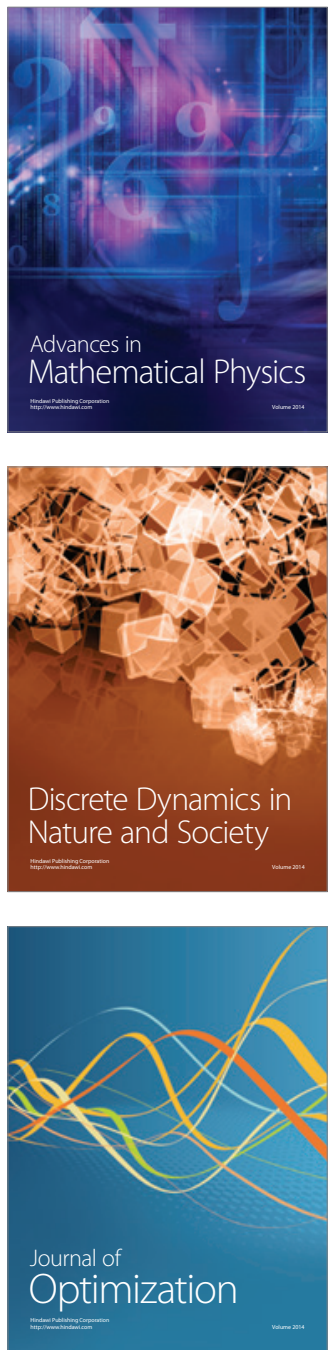